\title{
Pain Intensity and Pain Interference in Older Adults: Role of Gender, Obesity and High-Sensitivity C-Reactive Protein
}

\author{
Vahid Eslami ${ }^{\mathrm{a}, \mathrm{b}}$ Mindy J. Katz ${ }^{\mathrm{a}}$ Robert S. White ${ }^{\mathrm{a}}$ Erin Sundermann ${ }^{\mathrm{a}}$ \\ Julie M. Jiang ${ }^{\text {a }}$ Ali Ezzati $^{a}$ Richard B. Lipton ${ }^{a}$

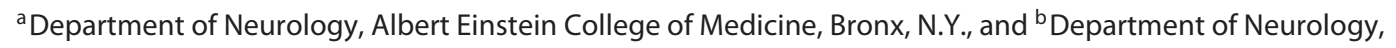 \\ Johns Hopkins University School of Medicine, Johns Hopkins Hospital, Baltimore, Md., USA
}

\section{Key Words}

Pain intensity · Pain interference $\cdot$ High-sensitive C-reactive protein · Body mass index · Gender

\begin{abstract}
Background: Among older adults, pain intensity and pain interference are more common in women than men and associated with obesity and inflammatory markers. Objective: We examined whether the obesity and pain relationship is mediated by the high-sensitivity C-reactive protein (hsCRP), a nonspecific marker of systemic inflammation, and whether this relationship differs by sex. Methods: Items from Medical Outcomes Study Short Form-36 were used to measure pain intensity and pain interference in daily life. Ordinal logistic regression was used to assess the cross-sectional association among body mass index (BMI), hsCRP levels, pain intensity and pain interference using gender-stratified models adjusted for demographic variables. Results: Participants included 667 community-residing adults over the age of 70 years, free of dementia, enrolled in the Einstein Aging Study (EAS). In women $(n=410)$, pain intensity was associated with obesity [BMI $\geq 30$ vs. normal, odds ratio $(\mathrm{OR})=2.29,95 \%$ confidence interval $(\mathrm{Cl}) 1.43-3.68]$ and higher hsCRP $(\mathrm{OR}=1.28,95 \% \mathrm{Cl} 1.08-1.51)$. In a model with
\end{abstract}

\section{KARGER}

๑ 2016 S. Karger AG, Basel

E-Mail karger@karger.com

www.karger.com/ger obesity and hsCRP, both remained significant, but the association between hsCRP and pain intensity was somewhat attenuated. Obesity $(\mathrm{OR}=3.04,95 \% \mathrm{Cl} 1.81-5.11)$ and higher hsCRP levels (OR $=1.30,95 \% \mathrm{Cl} 1.08-1.56)$ were also independently associated with greater pain interference in women. After adjustment for pain intensity and BMI, hsCRP was no longer associated with pain interference in women. Greater pain intensity and being overweight or obese continued to be significantly associated with pain interference in women. In men ( $n=257)$, obesity and hsCRP were not associated with pain intensity or pain interference. Conclusions: In women, the relationship between obesity and higher levels of pain intensity or interference may be accounted for by factors related to hsCRP.

๑ 2016 S. Karger AG, Basel

\section{Introduction}

About one fifth of Americans aged 65 years or older report a pain-related problem that persisted for more than $24 \mathrm{~h}$ in the past 30 days, and $17.5 \%$ use a prescription analgesic $[1,2]$. Pain interferes with activities of daily living in about $60 \%$ of older adults who reported any pain in the last month [3]. The prevalence of chronic pain is

Richard B. Lipton, MD

Saul B. Korey Department of Neurology

Albert Einstein College of Medicine

1300 Morris Park Avenue, Van Etten 3C12, Bronx, NY 10461 (USA)

E-Mail Richard.Lipton@einstein.yu.edu 
higher in older than in younger adults, though it is not clear if the prevalence increases with age above age 70 years [4]. Pain is commonly measured by separately assessing pain intensity and its functional consequences (pain interference) $[5,6]$. Pain interference as reflected by measures of activity limitations and health-related quality of life increases with age [3]. The relationship between pain intensity and pain interference can be characterized by a threshold effect where low levels of pain intensity are rarely associated with pain interference; however, moderate to severe levels of pain intensity are often associated with pain interference $[6,7]$.

Risk factors for pain in older adults include female gender [8], obesity [9], and biomarkers of inflammation [10]. Obesity is characterized by systemic inflammation and reflected in elevated levels of inflammatory biomarkers, including high-sensitivity C-reactive protein (hsCRP) $[11,12]$. Elevated levels of systemic inflammation may increase the likelihood of pain by decreasing pain thresholds [13-15]. Therefore, it is important to evaluate the potential pathways that link obesity and systemic inflammation to pain intensity and pain interference.

Compared to men, women are twice as likely to experience chronic pain $[4,16,17]$. Therefore, we chose to examine the role of obesity and inflammation as risk factors for pain separately in women and men. We hypothesized that the association of obesity, as measured by body mass index (BMI), with pain intensity and pain interference may be mediated through systemic inflammation. We further hypothesized that the influence of inflammation, as measured by hsCRP, on pain interference is accounted for by its effect on pain intensity.

\section{Methods}

\section{Subjects}

Our sample consisted of participants from the Einstein Aging Study (EAS), a community-based, longitudinal study of adults aged 70 years and older. Participants were systematically recruited from Bronx County, N.Y., using Medicare eligibility information and voter registration lists. EAS exclusionary criteria included being non-English speaking, nonambulatory, having visual or auditory sensory impairment, or any other condition that would impede assessment.

The sample consisted of individuals who completed an annual EAS clinic visit and had data for pain $(\mathrm{n}=770)$. They were required to have data on hsCRP and BMI $(\mathrm{n}=709)$ for the corresponding clinic visit, criteria met by $92 \%$ of the initial participants with available data for pain. The general characteristics of 61 participants with missing data on HsCRP or BMI were not different from the final sample. After excluding persons with dementia $(n=29)$, and those with BMI $<18.5(n=13)$, our final sample consisted of 667 participants. EAS study design, enrollment procedures, and data collection have been previously described $[18,19]$.

Written informed consent was obtained during all clinic visits in accordance with study protocols approved by the Committee on Clinical Investigation, the Institutional Review Board of the Albert Einstein College of Medicine.

\section{Clinical Evaluation}

Annual clinic visits at the EAS Clinic involved assessments of sociodemographic characteristics (age, sex, race, and education), medical history, current medical status, and a neurological exam. BMI was calculated as weight in kilograms divided by the square of height in meters. Participants were categorized into four groups based on BMI: underweight $(\mathrm{BMI}<18.5)$, normal weight $(\mathrm{BMI}=$ 18.5-24.9), overweight (BMI $=25-29.9)$, and obese $(\mathrm{BMI} \geq 30)[4$, $15,20]$. Underweight individuals were excluded from analyses because there were too few individuals in this category. The normal weight category was used as the reference in all models. Waist circumference was measured at the level of the umbilicus.

\section{Blood Sampling}

A phlebotomist drew $20 \mathrm{ml}$ whole blood samples from participants. Blood was collected in red top tubes without containing any coagulant and was centrifuged at $3,000 \mathrm{~g}$ for $10 \mathrm{~min}$ at $4^{\circ} \mathrm{C}$. Blood samples were processed in batches at the Albert Einstein College of Medicine General Clinical Research Center Core Laboratory using the same CRP Latex reagent on Beckman Coulter AU analyzers according to established procedures. In our study, we collected samples in sterile tubes, chilled, and processed them quickly to preserve inflammatory stability according to established procedures recommended by Beckman Coulter and the current literature $[21,22]$.

We used the serum for analysis of the hsCRP throughout the EAS longitudinal study so that continued use ensures consistency and comparability. HsCRP was measured using the CRP Ultra Wide Range Reagent Kit (Equal Diagnostics Inc., Exton, Pa., USA), a latex-enhanced turbid metric immunoassay with a sensitivity range from 0.05 to $160.0 \mathrm{mg} / \mathrm{dl}$. The interassay coefficient of variation was $<6 \%$.

\section{Pain Evaluation}

We assessed pain intensity and pain interference using the bodily pain subscale from a self-report, quality of life questionnaire, the Medical Outcomes Study Short Form-36 (MOS SF-36). Reliability and validity for the MOS SF-36 have been previously demonstrated in an elderly population $[23,24]$. The pain intensity question asked: 'How much bodily pain have you had during the past 4 weeks?' Scores ranged from 1 to 6 with $1=$ 'none' and $6=$ 'very severe'. Based on this score, participants were categorized into three pain intensity groups: no pain (score $=1$ ), mild pain (score $=2-3)$, and moderate/severe pain $($ score $=4-6)$. The pain interference question asked: 'During the past 4 weeks, how much did pain interfere with your normal work, including both work outside the home and housework?' Scores ranged from 1 to 5 with $1=$ 'not at all' and $5=$ 'extremely'. Based on this score, participants were categorized into three pain interference groups: no interference (score $=1)$, mild pain interference (score $=2)$, and moderate/ severe pain interference (scores $=3,4$, and 5). These methods of surveying and categorizing pain outcomes have been used in population-based studies previously $[25,26]$. 


\section{Statistical Analysis}

All analyses were adjusted for ethnicity, age, and education, and were conducted in the overall group and stratified by sex in order to determine whether older men and women differ in the pattern of association between obesity, hsCRP levels, and pain intensity and interference. Demographic and clinical characteristics were compared between the pain intensity (no pain intensity vs. mild vs. severe) and pain interference (none vs. mild vs. severe) groups separately using analysis of variance (ANOVA) for continuous variables and Pearson's $\chi^{2}$ test or Fisher's exact test for categorical variables. Nonparametric equivalents were used for variables that violated assumptions of normality. HsCRP distribution was skewed and was log-transformed for all analyses. The dependent measures of pain intensity and pain interference were modeled using ordinal logistic regression. Three models were created for the pain intensity outcome. Model 1 included demographic factors (age, race, and education) and hsCRP. Model 2 included demographic factors and BMI categories. Model 3 included demographic factors, hsCRP, and BMI categories together.

Models 4-10 were created for the pain interference outcome. Model 4 included demographic factors (age, race, and education) and hsCRP. Model 5 included demographic factors and BMI categories. Model 6 included demographic factors, hsCRP, and BMI together. Model 7 included demographic factors and pain intensity categories. Model 8 included model 7 plus hsCRP. Model 9 included model 7 plus BMI categories. Model 10 included model 7 plus BMI categories and hsCRP together. Statistical significance was assigned at an alpha level of 0.05 .

Both in men and women, models 1-6 were fit using ordinal logistic regression. In models $7-10$, the parallel line assumption test was violated, and, therefore, they were fit using a generalized ordinal logistic models that allowed for a relaxation of the parallel line assumption for violator variables, allowing it to be expressed as two separate odds ratios (OR), while maintaining model parsimony and meaning for all other variables. SPSS 22 (Chicago, Ill., USA) and the STATA software, version 12.1 (College Station, Tex., USA) were used for statistical analysis.

\section{Results}

Table 1 shows the demographic and clinical characteristics of the overall sample and stratified by sex and pain intensity status (no pain, mild pain, and moderate/severe pain intensity). Participants were predominately female (61.47\%), Caucasian (67.62\%), and had a mean age of 79.4 years $(\mathrm{SD}=5.49$; range $70-100$ years). In the overall sample, higher pain intensity levels were associated with higher hsCRP levels, higher BMI, and female sex. Among women, pain intensity levels were significantly associated with higher hsCRP levels and BMI. Conversely, among men, BMI and hsCRP levels did not differ between pain intensity groups. The association between BMI and hsCRP levels occurred in both men $(\mathrm{R}=0.14, \mathrm{p}=0.02)$ and women $(\mathrm{R}=0.32, \mathrm{p}<0.001)$.

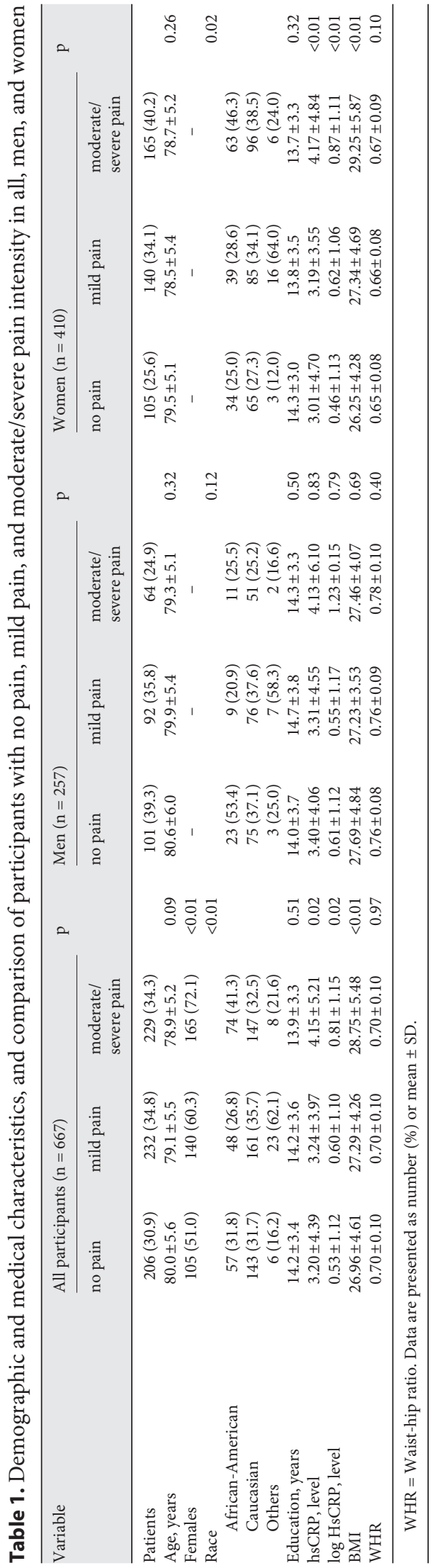

Gerontology 2017;63:3-12 DOI: $10.1159 / 000446651$ 
Table 2 displays and compares the demographic and clinical characteristics of the overall sample and stratified by sex and pain interference status (no pain, mild pain, and moderate/severe pain interference). Higher pain interference levels in the overall sample were significantly associated with higher hsCRP levels, BMI and the proportion of women. Among women, higher pain interference levels were significantly associated with higher hsCRP levels and BMI. Conversely, BMI and hsCRP levels did not differ between pain interference groups in men. The number of participants in each BMI group is shown in online supplementary table 1 (see www. karger.com/doi/10.1159/000446651 for all online suppl. material). The proportion of men and women within each pain intensity and pain interference category by hsCRP status (panels A and B) and BMI status (panels C and $D$ ) is displayed in online supplementary figure 1 . The results for women are presented in tables 3-5, and the results for men can be found in online supplementary tables 2-4.

\section{Ordinal Regression Models for Pain Intensity \\ Women}

Table 3 shows the results of ordinal logistic regression models $1-3$, which examined factors associated with higher pain intensity in women. Model 1 shows that the inflammatory marker hsCRP was significantly associated with pain intensity. The odds of higher pain intensity increased by $28 \%$ with each log unit increase of hsCRP $[\mathrm{OR}=1.28,95 \%$ confidence interval $(\mathrm{CI}) 1.08-1.51, \mathrm{p}=$ $0.004]$.

Model 2 shows that obese individuals $(B M I \geq 30)$ had 2.29 times greater risk for higher pain intensity compared with the normal BMI group ( $\mathrm{OR}=2.29,95 \% \mathrm{CI}$ $1.43-3.68, \mathrm{p}=0.001$ ); however, overweight subjects (BMI $=25-29.9$ ) were not at increased risk for higher pain intensity compared with the normal BMI group (OR = $1.10,95 \%$ CI $0.71-1.71, \mathrm{p}=0.66)$. Model 3 showed that when the hsCRP and BMI categories were combined in the same model, obesity continued to be significantly associated with greater pain intensity $(\mathrm{OR}=2.02,95 \% \mathrm{CI}$ 1.24-3.29, $\mathrm{p}=0.005)$; however, the association between pain intensity and hsCRP was attenuated and only marginally significant $(\mathrm{OR}=1.19,95 \%$ CI $1.00-1.42, \mathrm{p}=$ 0.05).

\section{Men}

Models 1-3 were repeated for men, and the results are shown in online supplementary table 2. Obesity and hsCRP were not associated with pain intensity.

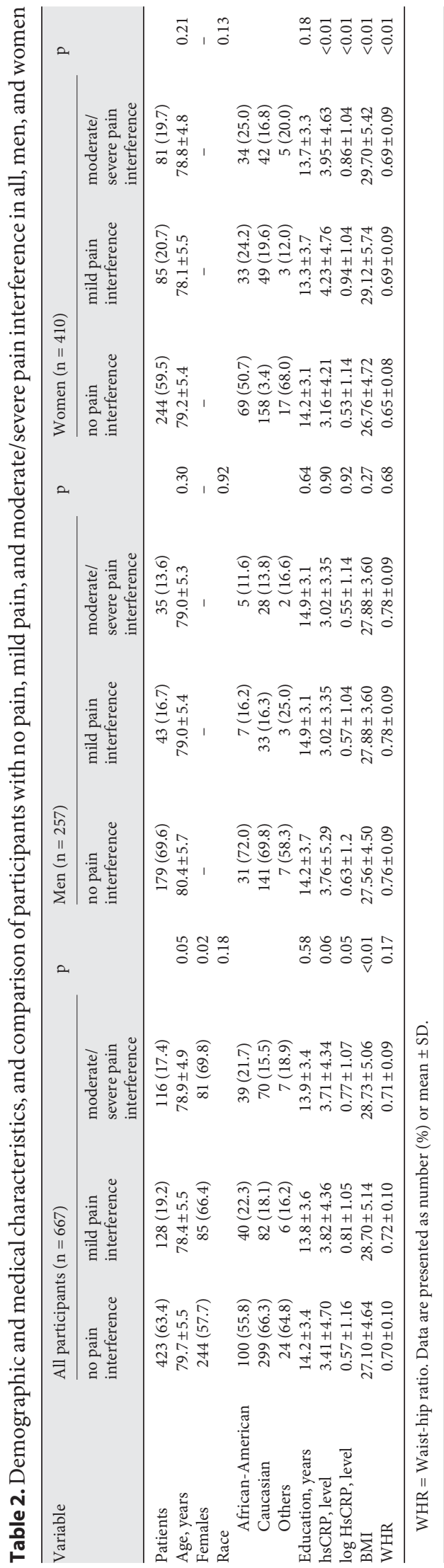

Eslami/Katz/White/Sundermann/Jiang/ Ezzati/Lipton
6

Gerontology 2017;63:3-12 DOI: $10.1159 / 000446651$ 
Table 3. Association of pain intensity level (none vs. mild vs. moderate-severe) with hsCRP and obesity in women: models of ordinal logistic regression adjusting for ethnicity, age, and education

\begin{tabular}{|c|c|c|c|c|c|c|c|c|c|}
\hline Variable & \multicolumn{3}{|c|}{ Model 1} & \multicolumn{3}{|c|}{ Model 2} & \multicolumn{3}{|c|}{ Model 3} \\
\hline Age (years) & 0.99 & $0.96-1.03$ & 0.40 & 0.99 & $0.96-1.03$ & 0.67 & 0.99 & $0.96-1.03$ & 0.72 \\
\hline Education (years) & 0.97 & $0.92-1.02$ & 0.22 & 0.97 & $0.92-1.02$ & 0.26 & 0.97 & $0.92-1.03$ & 0.30 \\
\hline log CRP (level) & 1.28 & $1.08-1.51$ & $<0.01$ & - & - & - & 1.19 & $1.00-1.42$ & 0.05 \\
\hline Overweight & - & - & - & 1.10 & $0.71-1.71$ & 0.66 & 1.06 & $0.68-1.65$ & 0.79 \\
\hline Obese and morbidly obese & - & - & - & 2.29 & $1.43-3.68$ & $<0.01$ & 2.02 & $1.24-3.29$ & $<0.01$ \\
\hline
\end{tabular}

Ethnicity is considered as all non-white participants vs. white participants.

Table 4. Association of pain interference level (none vs. mild vs. moderate-severe) with hsCRP and obesity in women: models of ordinal logistic regression adjusting for ethnicity, age, and education

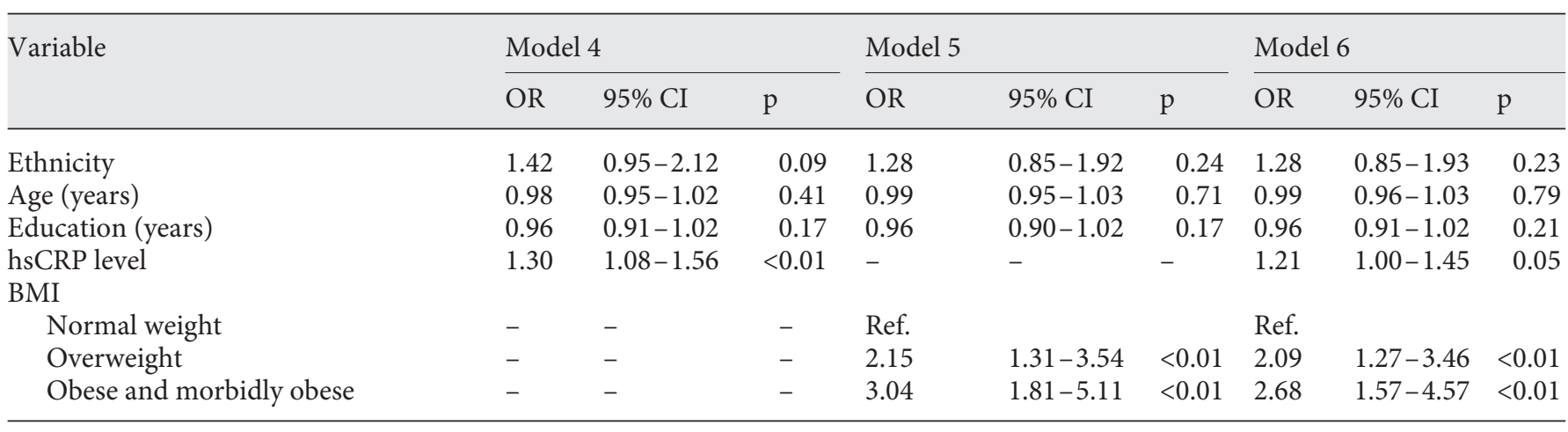

Ethnicity is considered as all non-white participants vs. white participants.

Table 5. Association of pain interference level (none vs. mild vs. moderate-severe) with obesity, log CRP level, and pain intensity in women: models of ordinal logistic regression adjusting for ethnicity, age, and education

\begin{tabular}{|c|c|c|c|c|c|c|c|c|c|c|c|c|}
\hline \multirow[t]{2}{*}{ Variable } & \multicolumn{3}{|c|}{ Model 7} & \multicolumn{3}{|c|}{ Model 8} & \multicolumn{3}{|c|}{ Model 9} & \multicolumn{3}{|c|}{ Model 10} \\
\hline & OR & $95 \%$ CI & $\mathrm{p}$ & OR & $95 \% \mathrm{CI}$ & $\mathrm{p}$ & OR & $95 \% \mathrm{CI}$ & $\mathrm{p}$ & OR & $95 \% \mathrm{CI}$ & $\mathrm{p}$ \\
\hline Ethnicity & 1.51 & $0.96-2.38$ & 0.08 & 1.50 & $0.95-2.36$ & 0.08 & 1.39 & $0.87-2.21$ & 0.16 & 1.39 & $0.88-2.23$ & 0.16 \\
\hline Age (years) & 0.99 & $0.95-1.03$ & 0.52 & 0.99 & $0.95-1.04$ & 0.68 & 1.00 & $0.95-1.04$ & 0.82 & 1.00 & $0.95-1.04$ & 0.89 \\
\hline Education (years) & 0.97 & $0.91-1.03$ & 0.39 & 0.97 & $0.91-1.04$ & 0.44 & 0.97 & $0.91-1.04$ & 0.38 & 0.97 & $0.91-1.04$ & 0.42 \\
\hline hsCRP level & - & - & - & 1.17 & $0.95-1.43$ & 0.14 & - & - & - & 1.12 & $0.90-1.39$ & 0.30 \\
\hline \multicolumn{13}{|l|}{ BMI } \\
\hline Normal weight & - & - & - & - & - & - & Ref. & & & Ref. & & \\
\hline Overweight & - & - & - & - & - & - & 2.54 & $1.43-4.51$ & $<0.01$ & 2.51 & $1.41-4.46$ & $<0.01$ \\
\hline Obese and morbidly obese & - & - & - & - & - & - & 2.29 & $1.28-4.12$ & $<0.01$ & 2.13 & $1.17-3.89$ & $<0.01$ \\
\hline Pain intensity, mild & 16.29 & $4.88-54.35$ & $<0.01$ & 16.11 & $4.83-53.81$ & $<0.01$ & 17.06 & $5.07-57.42$ & $<0.01$ & 17.07 & $5.06-57.50$ & $<0.01$ \\
\hline Pain intensity, severe & 82.45 & $24.82-273.90$ & $<0.01$ & 79.01 & $23.75-262.83$ & $<0.01$ & 83.96 & $25.01-281.83$ & $<0.01$ & 82.45 & $24.54-276.98$ & $<0.01$ \\
\hline
\end{tabular}

Ethnicity is considered as all non-white participants vs. white participants. 


\section{Ordinal Regression Models for Pain Interference}

Women

Table 4 shows the results of ordinal logistic regression models 4-6, which examined the associations among $\mathrm{BMI}$, hsCRP levels and pain interference without adjustment for pain intensity in women. Model 4 shows that a higher level of hsCRP was associated with higher levels of pain interference $(\mathrm{OR}=1.30,95 \%$ CI 1.08-1.55, $\mathrm{p}=$ $0.004)$. Model 5 shows that higher levels of pain interference were associated with both being overweight $(\mathrm{OR}=$ $2.15,95 \%$ CI 1.31-3.54, $\mathrm{p}<0.001)$ and obese $(\mathrm{OR}=3.04$, $95 \%$ CI 1.81-5.11, $\mathrm{p}<0.001)$ versus being normal weight. Model 6 shows that when hsCRP and BMI category are combined in the same model, the association between hsCRP and pain interference was slightly attenuated and only marginally significant $(\mathrm{OR}=1.21,95 \% \mathrm{CI} 1.00-1.45$, $\mathrm{p}=0.05)$. The relationship between pain interference and being overweight $(\mathrm{OR}=2.09,95 \% \mathrm{CI} 1.27-3.46 ; \mathrm{p}=$ $0.001)$ or obese $(\mathrm{OR}=2.68,95 \% \mathrm{CI} 1.57-4.57, \mathrm{p}=0.001)$ versus normal weight was slightly attenuated by the addition of hsCRP to the model but continued to be strongly significant.

\section{Men}

Neither obesity nor hsCRP was associated with pain interference (online suppl. table 3: models 4-6).

\section{Ordinal Regression Models for Pain Interference \\ Including Pain Intensity as Variable \\ Women}

Table 5 (models 7-10) shows the results of ordinal logistic regression, examining factors associated with category of pain interference taking hsCRP, BMI category and pain intensity into account for women. In model 7, mild and moderate/severe pain intensity was associated with an increased risk of pain interference. Model 8 (adjusting for hsCRP and pain intensity) showed that hsCRP was not associated with pain interference, but pain intensity continued to be significantly associated with pain interference. In model 9 (adjusting for BMI and pain intensity), BMI and pain intensity were both strongly associated with pain interference. In model 10 (adjusting for BMI, pain intensity, and hsCRP), BMI and pain intensity were both significantly associated with pain interference, while hsCRP was not significant in any of these models.

\section{Men}

Pain intensity was associated with higher pain interference; however, obesity and hsCRP were not associated with pain interference in men (online suppl. table 4: models 7-10).

Finally, we repeated our analysis using waist circumference rather than BMI in men and women. Findings showed virtually identical patterns. In men, waist circumference was not associated with pain intensity or interference. In women, hsCRP and waist circumference were independently associated with pain intensity and pain interference, and the association between hsCRP and pain interference was attenuated after adding waist circumference to both models of pain intensity and interference (results not shown).

\section{Discussion and Conclusions}

This cross-sectional study examined the association of BMI and hsCRP with pain intensity and pain interference. We will first discuss findings in women for pain intensity as the outcome and then for pain interference. We then consider the potential reasons for the lack of effects in men.

In women, after adjusting for demographic variables, hsCRP and obesity were associated with pain intensity and pain interference when examined separately. In a model including both hsCRP and obesity, the independent associations of each with pain intensity remained statistically significant but were modestly attenuated. This attenuation could reflect shared variance between measures of inflammation and BMI. Other studies have also found obesity to be associated with an increased incidence of pain in the elderly [4, 9, 27-29]. A previous study in the EAS by McCarthy et al. [4] reported that obesity was associated with a twofold increased risk of chronic pain, while morbid obesity (BMI $\geq 35$ ) was associated with fourfold increase in the risk of chronic pain. In a longitudinal study, the overall prevalence of pain in older adults was $32.7 \%$ at baseline, and this estimate increased significantly with higher quartiles of BMI or waist circumference. Being overweight at baseline was associated with an increased risk of pain in a 6-year follow-up period [27].

Our findings suggest that the influence of obesity on pain intensity may be mediated through the inflammatory processes indexed by hsCRP. Adipose tissue is an active endocrine organ which secretes substances to regulate several metabolic and physiological processes [30]. Since the relationship between obesity and pain is attenuated yet remains significant, it is possible that the obesity and pain relationship is mediated through other obesity- 
related inflammatory markers not measured in this study. Examples include adipocytokines, such as leptin, resistin, and adiponectin [31]. These cytokines are linked to obesity and may contribute to pain and inflammation independent of hsCRP $[31,32]$.

The hsCRP is a useful marker of systemic inflammation that has been linked to many health conditions such as cardiovascular disease, myocardial infarction, diabetes, and osteoporosis [33]. While hsCRP levels of more than $10 \mathrm{mg} / \mathrm{l}$ reflect clinically significant inflammation, subclinical levels of it (3-10 mg/l) suggest an elevated risk for the development of disease [34]. High hsCRP levels have been associated with a number of pain conditions such as fibromyalgia and rheumatoid arthritis [33]. A pain study in females that used cold pain sensitivity ratings showed the direct association between CRP and pain threshold and tolerance after adjusting for age, BMI, time to reach pain threshold or tolerance, and clinical pain status [35].

In addition, we showed that both higher hsCRP levels and being overweight or obese were associated with greater pain interference in women, in separate models. Similar to our finding with pain intensity, the relationships between pain interference with both BMI and hsCRP remained statistically significant but were modestly attenuated when hsCRP and BMI were included in the same model. The attenuation of the relationship between BMI and pain interference by the addition of hsCRP to the model suggests that the association between BMI and pain interference may be mediated, at least in part, by hsCRP. To date, there are limited studies that examined pain interference in older adults $[26,36]$. A longitudinal study has reported that in comparison with normal weight, being overweight or obese was associated with an increased risk of future development of pain interference. This association was stronger in obese individuals compared to overweight individuals, suggesting a dose-dependent effect of BMI on pain interference [28].

In our study, we modelled pain interference as the outcome, adding pain intensity to the measures of demographic factors, obesity, and inflammation discussed above. As expected, pain intensity was strongly associated with pain interference in both men and women. Among women, when pain intensity was included in the model, hsCRP was not significantly associated with pain interference; however, obesity remained significantly associated. To our knowledge, prior studies have not assessed the influence of inflammatory markers on pain interference in older adults.

Pain Intensity and Interference in Elderly
The precise mechanisms linking obesity, inflammation, pain intensity, and pain interference warrant further exploration. The cross-sectional design of this study precludes conclusions regarding causality or directionality of the relationships among obesity, hsCRP and pain. Obesity is a proinflammatory state characterized by the release of inflammatory compounds including hsCRP and insulin resistance-inducing substances from visceral adipose tissue $[29,37,38]$. Adipose tissue also releases factors which stimulate CRP production in the liver [20]. This gives biological plausibility to the idea that obesity results in hsCRP elevation, which, in turn, leads to a higher rate of pain.

We cannot rule out the possibility of reverse causality, whereby pain leads to reduced physical activity and/or overeating and, in turn, increased BMI $[29,39]$. It is also possible for pain and obesity to have a bidirectional relationship, whereby obesity leads to inflammation and pain and pain decreases activity leading to obesity. For example, obesity is a major risk factor for osteoarthritis, a highly prevalent pain disorder in the elderly [29]. Obesity causes joint damage through different mechanisms such as increased cytokines, the coexistence of obesity with metabolic impairments, and mechanical loading [31]. On the other hand, osteoarthritis limits mobility, which can lead to an increase in weight [40]. This could be suggestive of a bidirectional relationship leading to increased inflammation [31, 41, 42].

The associations among BMI, hsCRP and pain outcomes were found only in women, a finding consistent with prior demonstrations of sex difference [43]. In comparison with men, women report more frequent pain, higher pain intensity, more pain interference, and longer durations of pain [44]. Females generally report lower pain thresholds and tolerance than males. In a study of older Canadian adults, among participants with pain, women were more likely to experience interferences with physical and psychological functioning than men [36]. A longitudinal study in adults aged greater than 50 years found that the onset of pain interference increases with age and has a strong gender difference, with women reporting more pain interference [26]. This could reflect sex difference in response to pain, in the social rules governing pain reporting, or in biological mechanisms underlying pain [44].

We found that pain intensity and interference are related to obesity and inflammation in women and not men. There are a number of possible explanations for this sex difference. Women have more adipose tissue than men, and a higher percent body fat, at a given BMI [45]. 
Additionally, the association between adiposity and lowgrade systemic inflammation, as indexed by CRP level, is higher in women in comparison with men [46]. In our sample, the positive correlation between hsCRP and BMI was significant in both men and women but higher in women $(\mathrm{R}=0.32)$ compared to men $(\mathrm{R}=0.14)$. At a given BMI, women may have more adipose tissue than men. Alternatively, adipose tissue in women may produce more inflammatory cytokines than adipose tissue in men. In either case, greater levels of CRP in women may lead to more pain. Sex hormones offer another potential explanation. Older men have higher estradiol levels compared with age-matched women [47]. As estrogens have an inhibitory effect on inflammatory cytokines [48], higher estradiol levels in older men may protect against the proinflammatory influence of obesity.

Testosterone, similar to estrogen, has anti-inflammatory properties [49]. It reduces serum levels of the proinflammatory cytokines and increases the levels of the antiinflammatory cytokines [50, 51].

Although testosterone levels decrease in men with aging, it remains the predominant sex hormone in men throughout life [49]. Therefore, older men have higher levels of estrogen and testosterone, and possibly more anti-inflammatory properties compared to older women. Future investigations should examine the effect of hormone levels on inflammation, pain intensity, and interference.

This study has strengths and limitations. Strengths include the EAS population, which consists of relatively healthy, community-dwelling individuals who were systematically recruited from our Bronx community. We measured pain intensity and interference as well as hsCRP. The cross-sectional design precludes the determination of temporal sequence. Longitudinal studies examining the association between obesity and pain using inflammatory markers are needed. Because of the budgetary limitations, we examined a single inflammatory biomarker, hsCRP. The levels of other inflammatory markers that might contribute to the obesity-pain relationship were not assessed. Therefore, our results are limited to CRP, a nonspecific marker of systemic inflammation, and cannot be generalized to all types of inflammation $[11,12,15]$.

Serum samples may contain inflammatory cytokines secreted from leukocytes, mainly during the clotting process $[21,52]$. While cytokines regulate the production of hsCRP, this only takes place in the tissues, mainly liver $[21,53]$. Therefore, we are not sure if the release of cytokines during the serum production can impact the hsCRP levels in the blood tube. Based on some internal data analysis, no significant statistical difference between CRP levels in serum and plasma was reported [22].

In summary, we found that hsCRP, a nonspecific marker of systemic inflammation, plays a critical role in the relationship between obesity and pain; however, this role appears to be specific to older women and not men. In women, the relationship among obesity, hsCRP levels and pain may reflect a complex interaction of fat mass, joint inflammation, mild systemic inflammation, and pain.

\section{Acknowledgments}

The authors would like to thank all EAS staff for assistance with participant recruitment and the study participants who generously gave their time in support of this research.

This research was supported by National Institute on Aging Grants AG03949, AG026728, TL1RR000087, T32-GM007288, CTSA 1UL1TR001073 from the National Center for Advancing Translational Sciences (NCATS), the Leonard and Sylvia Marx Foundation, and the Czap Foundation.

\section{Disclosure Statement}

All authors declare that there are no financial, personal, or other potential conflicts of interest to report.

\section{References}

1 National Center for Health Statistics: Health, United States, 2006, with Chartbook on Trends in the Health of Americans. Hyattsville, National Center for Health Statistics, 2006.

2 National Center for Health Statistics: Health, United States, 2012, with Special Feature on Emergency Care. Hyattsville, National Center for Health Statistics, 2013.
- 3 Thomas E, Peat G, Harris L, Wilkie R, Croft PR: The prevalence of pain and pain interference in a general population of older adults: cross-sectional findings from the North Staffordshire Osteoarthritis Project (NorStOP). Pain 2004;110:361-368.

4 McCarthy LH, Bigal ME, Katz M, Derby C, Lipton RB: Chronic pain and obesity in elderly people: results from the Einstein Aging Study. J Am Geriatr Soc 2009;57:115-119. 
5 Johannes CB, Le TK, Zhou X, Johnston JA, Dworkin RH: The prevalence of chronic pain in United States adults: results of an Internetbased survey. J Pain 2010;11:1230-1239.

-6 Jensen MP, Smith DG, Ehde DM, Robinsin LR: Pain site and the effects of amputation pain: further clarification of the meaning of mild, moderate, and severe pain. Pain 2001; 91:317-322.

7 Arnstein P, Caudill M, Mandle CL, Norris A, Beasley R: Self efficacy as a mediator of the relationship between pain intensity, disability and depression in chronic pain patients. Pain 1999;80:483-491.

8 Leveille SG, Zhang Y, McMullen W, KellyHayes M, Felson DT: Sex differences in musculoskeletal pain in older adults. Pain 2005; 116:332-338.

-9 Shi Y, Hooten WM, Roberts RO, Warner DO: Modifiable risk factors for incidence of pain in older adults. Pain 2010;151:366-371.

10 Stannus OP, Jones G, Blizzard L, Cicuttini FM, Ding C: Associations between serum levels of inflammatory markers and change in knee pain over 5 years in older adults: a prospective cohort study. Ann Rheum Dis 2013; 72:535-540.

-11 Brooks GC, Blaha MJ, Blumenthal RS: Relation of C-reactive protein to abdominal adiposity. Am J Cardiol 2010;106:56-61.

12 Park HS, Park JY, Yu R: Relationship of obesity and visceral adiposity with serum concentrations of CRP, TNF-alpha and IL-6. Diabetes Res Clin Pract 2005;69:29-35.

-13 Schaible HG, von Banchet GS, Boettger MK, Brauer R, Gajda M, Richter F, Hensellek S, Brenn D, Natura G: The role of proinflammatory cytokines in the generation and maintenance of joint pain. Ann NY Acad Sci 2010; 1193:60-69.

14 Sommer C, Kress M: Recent findings on how proinflammatory cytokines cause pain: peripheral mechanisms in inflammatory and neuropathic hyperalgesia. Neurosci Lett 2004; 361:184-187.

15 Briggs MS, Givens DL, Schmitt LC, Taylor CA: Relations of C-reactive protein and obesity to the prevalence and the odds of reporting low back pain. Arch Phys Med Rehabil 2013;94:745-752.

16 Fillingim RB, King CD, Ribeiro-Dasilva MC, Rahim-Williams B, Riley JL 3rd: Sex, gender, and pain: a review of recent clinical and experimental findings. J Pain 2009;10:447-485.

-17 Hirsh AT, Dillworth TM, Ehde DM, Jensen MP: Sex differences in pain and psychological functioning in persons with limb loss. J Pain 2010;11:79-86.

18 Lipton RB, Katz MJ, Kuslansky G, Sliwinski MJ, Stewart WF, Verghese J, Crystal HA, Buschke H: Screening for dementia by telephone using the memory impairment screen. J Am Geriatr Soc 2003;51:1382-1390.
Katz MJ, Lipton RB, Hall CB, Zimmerman ME, Sanders AE, Verghese J, Dickson DW, Derby CA: Age-specific and sex-specific prevalence and incidence of mild cognitive impairment, dementia, and Alzheimer dementia in blacks and whites: a report from the Einstein Aging Study. Alzheimer Dis Assoc Disord 2012;26:335-343.

-20 Visser M, Bouter LM, McQuillan GM, Wener $\mathrm{MH}$, Harris TB: Elevated C-reactive protein levels in overweight and obese adults. JAMA 1999;282:2131-2135.

21 Zhou X, Fragala MS, McElhaney JE, Kuchel GA: Conceptual and methodological issues relevant to cytokine and inflammatory marker measurements in clinical research. Curr Opin Clin Nutr Metab Care 2010;13:541547.

22 Beckman Coulter Inc: Crp Methodology, 2016.

23 Peek MK, Ray L, Patel K, Stoebner-May D, Ottenbacher KJ: Reliability and validity of the SF-36 among older Mexican Americans. Gerontologist 2004;44:418-425.

24 Brazier JE, Walters SJ, Nicholl JP, Kohler B: Using the SF-36 and Euroqol on an elderly population. Qual Life Res 1996;5:195-204.

25 Blyth FM, March LM, Brnabic AJ, Jorm LR, Williamson M, Cousins MJ: Chronic pain in Australia: a prevalence study. Pain 2001;89: 127-134.

26 Thomas E, Mottram S, Peat G, Wilkie R, Croft $P$ : The effect of age on the onset of pain interference in a general population of older adults: prospective findings from the North Staffordshire Osteoarthritis Project (NorStOP). Pain 2007;129:21-27.

27 Heim N, Snijder MB, Deeg DJ, Seidell JC, Visser M: Obesity in older adults is associated with an increased prevalence and incidence of pain. Obesity 2008;16:2510-2517.

28 Jordan KP, Thomas E, Peat G, Wilkie R, Croft $P$ : Social risks for disabling pain in older people: a prospective study of individual and area characteristics. Pain 2008;137:652-661.

29 Ray L, Lipton RB, Zimmerman ME, Katz MJ, Derby CA: Mechanisms of association between obesity and chronic pain in the elderly. Pain 2011;152:53-59.

- 30 Uslu S, Kebapci N, Kara M, Bal C: Relationship between adipocytokines and cardiovascular risk factors in patients with type 2 diabetes mellitus. Exp Ther Med 2012;4:113120.

31 Sowers MR, Karvonen-Gutierrez CA: The evolving role of obesity in knee osteoarthritis. Curr Opin Rheumatol 2010;22:533-537.

- 32 Sugiura K, Tamakoshi K, Yatsuya H, Otsuka R, Wada K, Matsushita K, Kondo T, Hotta Y, Mitsuhashi H, Murohara T, Toyoshima H: Contribution of adipocytokines to low-grade inflammatory state as expressed by circulating C-reactive protein in Japanese men: comparison of leptin and adiponectin. Int J Cardiol 2008;130:159-164.
33 Lund Haheim L, Nafstad P, Olsen I, Schwarze $\mathrm{P}$, Ronningen KS: C-reactive protein variations for different chronic somatic disorders. Scand J Public Health 2009;37:640-646.

34 Kushner I, Rzewnicki D, Samols D: What does minor elevation of C-reactive protein signify? Am J Med 2006;119:166.e117-e128.

35 Afari N, Mostoufi S, Noonan C, Poeschla B, Succop A, Chopko L, Strachan E: C-reactive protein and pain sensitivity: findings from female twins. Ann Behav Med 2011;42:277283.

36 Scudds RJ, Ostbye T: Pain and pain-related interference with function in older Canadians: the Canadian Study of Health and Aging. Disabil Rehabil 2001;23:654-664.

37 Klein S, Allison DB, Heymsfield SB, Kelley DE, Leibel RL, Nonas C, Kahn R; Association for Weight Management and Obesity Prevention; NAASO, The Obesity Society; American Society for Nutrition; American Diabetes Association: Waist circumference and cardiometabolic risk: a consensus statement from Shaping America's Health: Association for Weight Management and Obesity Prevention; NAASO, The Obesity Society; the American Society for Nutrition; and the American Diabetes Association. Am J Clin Nutr 2007; 85:1197-1202.

38 Bray GA, Bellanger T: Epidemiology, trends, and morbidities of obesity and the metabolic syndrome. Endocrine 2006;29:109-117.

39 Somers TJ, Wren AA, Keefe FJ: Understanding chronic pain in older adults: abdominal fat is where it is at. Pain 2011;152:8-9.

40 McDonough CM, Jette AM: The contribution of osteoarthritis to functional limitations and disability. Clin Geriatr Med 2010;26:387-399.

41 Peat G, McCarney R, Croft P: Knee pain and osteoarthritis in older adults: a review of community burden and current use of primary health care. Ann Rheum Dis 2001;60:91-97.

42 Kraus VB, Stabler TV, Luta G, Renner JB, Dragomir $\mathrm{AD}$, Jordan JM: Interpretation of serum C-reactive protein (CRP) levels for cardiovascular disease risk is complicated by race, pulmonary disease, body mass index, gender, and osteoarthritis. Osteoarthritis Cartilage 2007;15:966-971.

43 Paller CJ, Campbell CM, Edwards RR, Dobs AS: Sex-based differences in pain perception and treatment. Pain Med 2009;10:289-299.

44 Dao TT, LeResche L: Gender differences in pain. J Orofac Pain 2000;14:169-184; discussion 184-195.

$\longrightarrow 45$ Gallagher D, Visser M, Sepulveda D, Pierson RN, Harris T, Heymsfield SB: How useful is body mass index for comparison of body fatness across age, sex, and ethnic groups? Am J Epidemiol 1996;143:228-239.

46 Thorand B, Baumert J, Doring A, Herder C, Kolb H, Rathmann W, Giani G, Koenig W, Group K: Sex differences in the relation of body composition to markers of inflammation. Atherosclerosis 2006;184:216-224. 
$\checkmark 47$ Yasui T, Matsui S, Tani A, Kunimi K, Yamamoto S, Irahara M: Androgen in postmenopausal women. J Med Invest 2012;59: $12-27$.

-48 Bruun JM, Nielsen CB, Pedersen SB, Flyvbjerg A, Richelsen B: Estrogen reduces pro-inflammatory cytokines in rodent adipose tissue: studies in vivo and in vitro. Horm Metab Res 2003;35:142-146.

49 Maggio M, Basaria S, Ceda GP, Ble A, Ling SM, Bandinelli S, Valenti G, Ferrucci L: The relationship between testosterone and molecular markers of inflammation in older men. J Endocrinol Invest 2005;28:116-119.

-50 Jones RD, Nettleship JE, Kapoor D, Jones HT, Channer KS: Testosterone and atherosclerosis in aging men: purported association and clinical implications. Am J Cardiovasc Drugs 2005;5:141-154.

51 Nettleship JE, Pugh PJ, Channer KS, Jones T, Jones RD: Inverse relationship between serum levels of interleukin-1beta and testoster- one in men with stable coronary artery disease. Horm Metab Res 2007;39:366-371.

52 Cannon JG, van der Meer JW, Kwiatkowski D, Endres S, Lonnemann G, Burke JF, Dinarello CA: Interleukin-1 beta in human plasma: optimization of blood collection, plasma extraction, and radioimmunoassay methods. Lymphokine Res 1988;7:457-467.

53 Yeh ET: A new perspective on the biology of C-reactive protein. Circ Res 2005;97:609611 